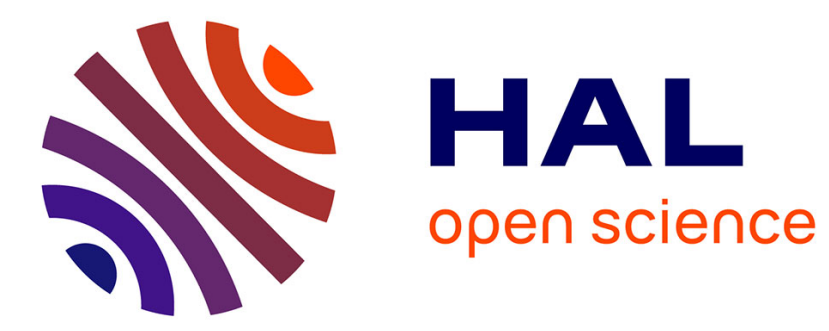

\title{
Optical Control of the Resonant Dipole-Dipole Interaction between Rydberg Atoms
}

\author{
Sylvain de Léséleuc, Daniel Barredo, Vincent Lienhard, Antoine Browaeys, \\ Thierry Lahaye
}

\section{- To cite this version:}

Sylvain de Léséleuc, Daniel Barredo, Vincent Lienhard, Antoine Browaeys, Thierry Lahaye. Optical Control of the Resonant Dipole-Dipole Interaction between Rydberg Atoms. Physical Review Letters, 2017, 119 (5), pp.053202 10.1103/PhysRevLett.119.053202 . hal-01592628

\section{HAL Id: hal-01592628 \\ https://hal.science/hal-01592628}

Submitted on 6 Oct 2017

HAL is a multi-disciplinary open access archive for the deposit and dissemination of scientific research documents, whether they are published or not. The documents may come from teaching and research institutions in France or abroad, or from public or private research centers.
L'archive ouverte pluridisciplinaire HAL, est destinée au dépôt et à la diffusion de documents scientifiques de niveau recherche, publiés ou non, émanant des établissements d'enseignement et de recherche français ou étrangers, des laboratoires publics ou privés. 


\title{
Optical Control of the Resonant Dipole-Dipole Interaction between Rydberg Atoms
}

\author{
Sylvain de Léséleuc, Daniel Barredo, Vincent Lienhard, Antoine Browaeys, and Thierry Lahaye \\ Laboratoire Charles Fabry, UMR 8501, Institut d'Optique, \\ CNRS, Univ Paris Sud 11, 2 Avenue Augustin Fresnel, 91127 Palaiseau cedex, France
}

(Received 9 May 2017; published 2 August 2017)

\begin{abstract}
We report on the local control of the transition frequency of a spin 1/2 encoded in two Rydberg levels of an individual atom by applying a state-selective light shift using an addressing beam. With this tool, we first study the spectrum of an elementary system of two spins, tuning it from a nonresonant to a resonant regime, where "bright" (super-radiant) and "dark" (subradiant) states emerge. We observe the collective enhancement of the microwave coupling to the bright state. We then show that after preparing an initial single spin excitation and letting it hop due to the spin-exchange interaction, we can freeze the dynamics at will with the addressing laser, while preserving the coherence of the system. In the context of quantum simulation, this scheme opens exciting prospects for engineering inhomogeneous $X Y$ spin Hamiltonians or preparing spin-imbalanced initial states.
\end{abstract}

DOI: 10.1103/PhysRevLett.119.053202

Real-world magnetic materials are often modeled with simple spin Hamiltonians exhibiting the key properties under study. Despite their simplified character, these models remain challenging to solve, and an actively explored approach is to implement them in pristine experimental platforms [1]. Such quantum simulators usually require an ordered assembly of interacting spins, also called qubits in the case of spin $1 / 2$, manipulated by global and local coherent operations. Local operations are a crucial element of a quantum simulator and they have been used, for example, to perform one-qubit rotations for quantum state tomography [2], to engineer two-qubit quantum gates (see, e.g., $[3,4]$ ), or to prepare peculiar initial states $[5,6]$ and apply local noise [7] for studies of many-body localization. To achieve a local operation, one usually shifts the frequency of one targeted qubit in the system. Depending on the physical platform, different approaches are used to accomplish this, such as applying static electric fields for quantum dots [8], or magnetic fluxes for superconducting circuits [9]. In atomic systems, focusing an offresonant laser beam on a single site can be used to apply an ac-Stark shift on ground-state levels [10-13].

Another promising approach for quantum information science and quantum simulation of spin Hamiltonians is atomic platforms based on Rydberg states [14,15], as they provide strong, tunable dipole-dipole interactions [16-18]. In addition, arrays of optical tweezers allow the efficient preparation of assemblies of up to 50 atoms, arranged in arbitrary geometries, as has been recently demonstrated $[19,20]$. One can encode a spin $1 / 2$ between the ground state and a Rydberg level, use the van der Waals interactions between two identical Rydberg states, and map the system onto an Ising-like Hamiltonian [21]. In this case, the spins can be manipulated globally by a resonant laser field and local addressing has been demonstrated using a far red-detuned focused laser beam shifting the ground-state energy of a particular atom in the ensemble [11].

In addition to the Ising Hamiltonian, the long-range $X Y$ Hamiltonian [22-27] can naturally be implemented with Rydberg atoms by using the dipolar spin-exchange interaction [28-32]. For principal quantum number $n \sim 60$, the direct dipole-dipole coupling $U=C_{3} / R^{3}$ between two atoms in Rydberg levels with orbital angular momentum differing by \pm 1 ensures strong interaction energies in the $1-10 \mathrm{MHz}$ range for atoms separated by $\sim 10 \mu \mathrm{m}$. The spin $1 / 2$ is in this case encoded in two Rydberg levels with a lifetime of a few $100 \mu$ s and a transition frequency in the microwave domain. Because of the exaggerated electric dipole of Rydberg states, high Rabi frequencies are obtained with low microwave power, resulting in a fast coherent manipulation of single atoms. However, for local tuning of the frequency, the previous schemes, shifting a ground-state level [11], are irrelevant, and the implementation of a selective Rydberg level shift was so far missing in the toolbox of quantum simulation of $X Y$ Hamiltonians.

In this Letter, using the Rydberg states $|\uparrow\rangle=|n D\rangle$ and $|\downarrow\rangle=\left|n^{\prime} P\right\rangle$ of ${ }^{87} \mathrm{Rb}$ separated by an energy $\hbar \omega_{0}$ to encode a spin $1 / 2$, we demonstrate selective addressing by using a focused addressing laser beam at $1005 \mathrm{~nm}$ [see Fig. 1(a)] to induce a controllable light-shift $\Delta \omega_{0}$ on $|\uparrow\rangle$, while the state $|\downarrow\rangle$ is unaffected. We illustrate this local tuning in a minimal system of two atoms separated by a distance $R$ and governed by the Hamiltonian

$$
H=H_{0}+\hbar \Delta \omega_{0} \frac{\sigma_{1}^{z}+1}{2}+U\left(\sigma_{1}^{+} \sigma_{2}^{-}+\sigma_{1}^{-} \sigma_{2}^{+}\right) .
$$

Here, $H_{0}=\hbar \omega_{0}\left(\sigma_{1}^{z}+\sigma_{2}^{z}\right) / 2$ is the single-atom Hamiltonian, $U=C_{3} / R^{3}$ is the strength of the dipole-dipole interaction, and $\sigma_{i}^{+}, \sigma_{i}^{-}$, and $\sigma_{i}^{z}$ denote the spin matrices 


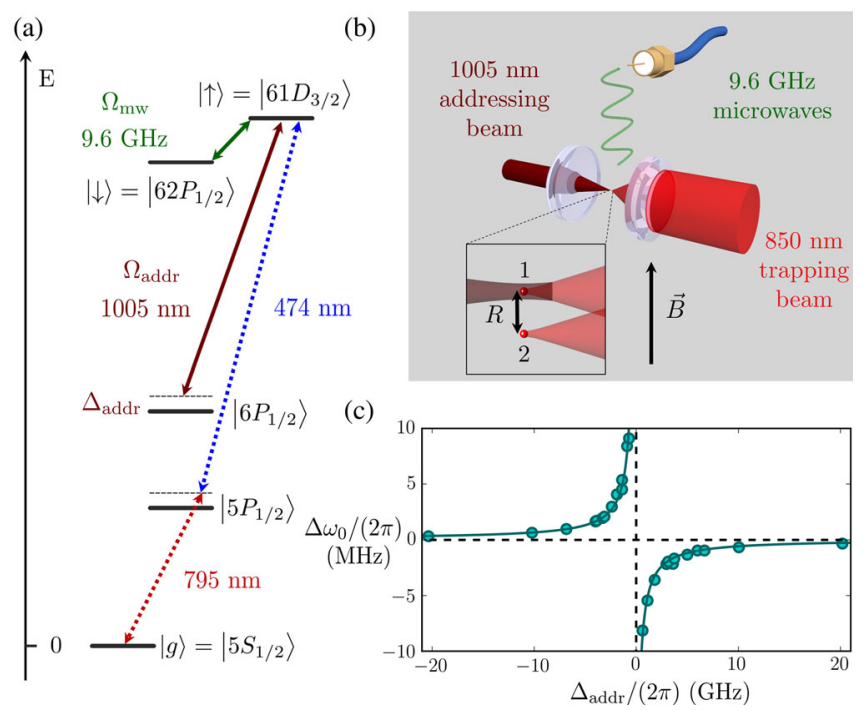

FIG. 1. (a) Scheme of the levels relevant for the experiment. The spin-1/2 states are encoded in two Rydberg levels. Microwaves couple $|\uparrow\rangle$ to $|\downarrow\rangle$ with Rabi frequency $\Omega_{\mathrm{mw}}$. The addressing beam couples $|\uparrow\rangle$ with $\left|6 P_{1 / 2}\right\rangle$ off-resonantly to induce a local light shift $\Delta \omega_{0}$. (b) Experimental setup. Two atoms separated by a distance $R$ and aligned along the quantization axis, defined by a $B=7 \mathrm{G}$ magnetic field. The $1005 \mathrm{~nm}$ addressing beam is focused on a single atom. (c) Light shift $\Delta \omega_{0}$ of state $|\uparrow\rangle$ measured by microwave spectroscopy, as a function of the detuning $\Delta_{\text {addr }}$ of the addressing laser. The full line represents the parameter-free expected light shift $\Delta \omega_{0}=\Omega_{\text {addr }}^{2} / 4 \Delta_{\text {addr }}$ with the calculated Rabi frequency $\Omega_{\mathrm{addr}} /(2 \pi)=158 \mathrm{MHz}$. Error bars are smaller than the symbol size.

acting on atom $i=1,2$. We neglect van der Waals interactions as, for the parameters of the experiments reported here, they are negligible with respect to all other energy scales [33]. We first perform microwave spectroscopy on this interacting two-atom system for various addressing light shifts $\Delta \omega_{0}$ on atom 1 . We show that the two atoms can be brought in and out of the resonant dipoledipole regime in a controlled way. Notably, in the resonant regime, we observe the collectively enhanced microwave coupling by a factor $\sqrt{2}$ between the state $|\uparrow \uparrow\rangle$ and the bright or super-radiant, superposition of the two atoms $(1 / \sqrt{2})(|\uparrow \downarrow\rangle+|\downarrow \uparrow\rangle)$ as well as the Rydberg blockade inhibiting the spin flip of the two atoms [31]. We then demonstrate that the spin-exchange dynamics observed after preparing the initial state $|\uparrow \downarrow\rangle$ can be stopped for a controlled amount of time. A dynamical phase is then accumulated by the addressed atom, due to the energy shift $\hbar \Delta \omega_{0}$, giving access to various two-atom entangled states. We show that coherence is maintained by letting the spin exchange resume. Finally, we discuss the possible limitations of this new tool for future quantum simulation experiments.

The experimental setup, shown schematically in Fig. 1(b), is described in detail in Refs. [21,34]. Briefly, we focus a red-detuned dipole trap beam with an aspheric lens $(\mathrm{NA}=0.5)$ into a magneto-optical trap of ${ }^{87} \mathrm{Rb}$ atoms, to a waist of approximately $1.1 \mu \mathrm{m}$. Multiple traps at arbitrary distances are created by imprinting an appropriate phase on the dipole trap beam $(850 \mathrm{~nm})$ with a spatial light modulator prior to focusing [35]. Single atoms are loaded in the desired traps by active sorting [19]. The temperature of the trapped single atoms is approximately $30 \mu \mathrm{K}$. An external magnetic field of $7 \mathrm{G}$ in the vertical direction defines the quantization axis.

We choose the Rydberg levels $|\uparrow\rangle=\mid 61 D_{3 / 2}$, $\left.m_{j}=3 / 2\right\rangle$ and $|\downarrow\rangle=\left|62 P_{1 / 2}, m_{j}=1 / 2\right\rangle$ to define the spin $1 / 2$ [see Fig. 1(a)]. The dipole-dipole coupling between $|\uparrow \downarrow\rangle$ and $|\downarrow \uparrow\rangle$ is $U=C_{3} / R^{3}$ with a calculated $C_{3}=h \times 7456 \mathrm{MHz} \cdot \mu \mathrm{m}^{3}$ [36,37] for this choice of Rydberg states. The atoms are excited from the ground state $|g\rangle=\left|5 S_{1 / 2}, F=2, m_{F}=2\right\rangle$ to $|\uparrow\rangle$ with a twophoton transition (wavelengths 795 and $474 \mathrm{~nm}$; the detuning from the intermediate state $\mid 5 P_{1 / 2}, F=2$, $\left.m_{F}=2\right\rangle$ is $740 \mathrm{MHz}$ ) of effective Rabi frequency $4 \mathrm{MHz}$. The spin-flip transition $|\uparrow\rangle \leftrightarrow|\downarrow\rangle$ is driven by a resonant microwave pulse at frequency $\omega_{0} /(2 \pi) \simeq$ $9.600 \mathrm{GHz}$ emitted by a dipole antenna placed outside the vacuum chamber. At the end of the experiment, a Rydberg deexcitation pulse (using the same lasers as for Rydberg excitation) transfers back atoms in $|\uparrow\rangle$ to the ground state and leaves atoms in $|\downarrow\rangle$ unaffected, which allows selective detection of $|\uparrow\rangle$ and $|\downarrow\rangle$.

For selective addressing, we use a laser beam at $1005 \mathrm{~nm}$, slightly detuned by a quantity $\Delta_{\text {addr }}$ from the transition $\left|6 P_{1 / 2}\right\rangle \leftrightarrow\left|n D_{3 / 2}\right\rangle$ [see Fig. 1(a)], which induces a light shift on $|\uparrow\rangle$, while $|\downarrow\rangle$ is not affected due to the electric dipole selection rules. Initially suggested in [38], this scheme was used for magic trapping of ground and Rydberg atoms [39]. The addressing beam from a cw Ti:sapphire laser is focused on trap 1 with a linear polarization perpendicular to the quantization axis. We choose a $3.4 \mu \mathrm{m}$ waist [40] as a tradeoff between adjacent site cross-talk ( $1 \%$ residual light shift at $R=5.2 \mu \mathrm{m}$ ) and alignment issues. The addressing laser is switched on and off by an electro-optic modulator with a rise time of $10 \mathrm{~ns}$. The laser frequency is locked on a commercial wavelength meter [42] to prevent long term drifts of $\Delta_{\text {addr }}$. The $\sigma^{+}$ polarization component of the addressing beam couples $\left|6 P_{1 / 2}, F=2, m_{F}=2\right\rangle$ with the Rydberg state $|\uparrow\rangle$; see Fig. 1(a). The Rabi frequency is calculated [36] to be $\Omega_{\text {addr }} /(2 \pi)=158 \mathrm{MHz}$ for an incident power $P=30 \mathrm{~mW}$ (only half of the linearly polarized laser power contributes). For a large detuning $\Delta_{\text {addr }} \gg \Omega_{\text {addr }}$, the state $|\uparrow\rangle$ experiences an ac-Stark shift $\Delta \omega_{0}=\Omega_{\text {addr }}^{2} / 4 \Delta_{\text {addr }}$, while the other state $|\downarrow\rangle$ remains unaffected by this laser (except for a calculated $\sim 4 \mathrm{MHz}$ ponderomotive light shift common on both states $[38,43])$.

In Fig. 1(c) we present the shifted qubit transition energy $\hbar\left(\omega_{0}+\Delta \omega_{0}\right)$ measured by microwave spectroscopy 
(driving the spin-flip transition) for different detuning $\Delta_{\text {addr }}$ of the addressing beam. The data are in excellent agreement with the expected light shift using the calculated Rabi frequency and where only the $\left|6 P_{1 / 2}\right\rangle \leftrightarrow\left|61 D_{3 / 2}\right\rangle$ transition frequency is a free parameter and is measured at 298.139 450 THz. This differential light shift between the two Rydberg states $|\uparrow\rangle$ and $|\downarrow\rangle$ allows one to tune the spinflip transition frequency of atom 1 and bring it in and out of resonance with atom 2 .

The effect of the dipole-dipole interaction between two atoms can now be controlled with the addressing beam. We first recall the two-atom energy spectrum in the presence of the dipole-dipole interaction $U$ and the addressing light shift $\hbar \Delta \omega_{0}$ as illustrated in Fig. 2(a). Without the addressing

(a)

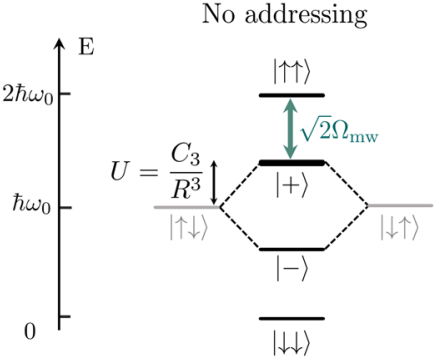

Addressing on atom 1

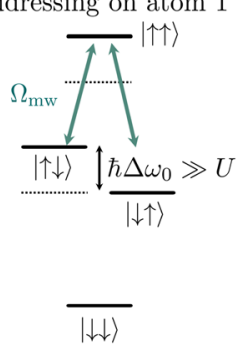

(b)
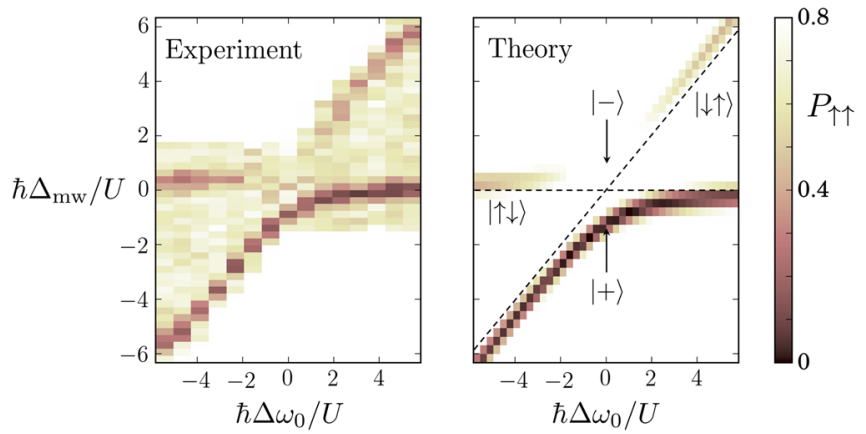

(c)
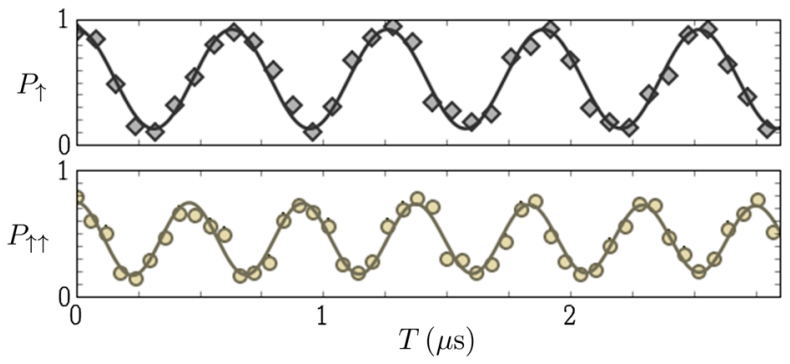

FIG. 2. (a) Two-atom energy structure in the resonant (left) and off-resonant (right) limit. (b) Microwave spectroscopy for $U / h=$ $0.40 \mathrm{MHz} \quad(R=25 \mu \mathrm{m})$ and $\Omega_{\mathrm{mw}} /(2 \pi)=0.1 \mathrm{MHz}$ starting from $|\uparrow \uparrow\rangle$. At resonance, the dark state is not coupled anymore by the microwave field. (c) Rabi oscillation for $U / h=4.09 \mathrm{MHz}$ $(R=12 \mu \mathrm{m})$ driven by microwaves with a Rabi frequency $\Omega_{\mathrm{mw}} /(2 \pi)=1.6 \mathrm{MHz}$. Upper panel: driving the single atom transition $|\uparrow\rangle \leftrightarrow|\downarrow\rangle$ at $\Delta_{\mathrm{mw}}=0$. Lower panel: observing the enhanced microwave coupling on the transition $|\uparrow \uparrow\rangle \leftrightarrow|+\rangle$ at $\Delta_{\mathrm{mw}}=-U$. Error bars are smaller than the symbol size. laser, the dipole-dipole interaction lifts the degeneracy between $|\uparrow \downarrow\rangle$ and $|\downarrow \uparrow\rangle$ and the Hamiltonian eigenstates become $| \pm\rangle=(1 / \sqrt{2})(|\uparrow \downarrow\rangle \pm|\downarrow \uparrow\rangle)$ separated in energy by $2 U=2 C_{3} / R^{3}$. With the addressing laser focused on the first atom, the state $|\uparrow \downarrow\rangle$ is shifted by an energy $\hbar \Delta \omega_{0}$ and is not resonant anymore with $|\downarrow \uparrow\rangle$. A microwave field at angular frequency $\omega=\omega_{0}+\Delta_{\text {mw }}$ drives single spin-flip transitions with Rabi frequency $\Omega_{\mathrm{mw}}$ to probe the system. At resonance $\left(\Delta \omega_{0}=0\right)$, the microwave coupling between the super-radiant state $|+\rangle$ and $|\uparrow \uparrow\rangle$ is enhanced to $\sqrt{2} \Omega_{\mathrm{mw}}$ while it vanishes for the subradiant state $|-\rangle$. In the limit of strong addressing $\hbar \Delta \omega_{0} \gg U$, the dipole-dipole interaction can be neglected and the microwave field is expected to couple equally $|\uparrow \uparrow\rangle$ to the two single-flipped spin states.

To study the system by microwave spectroscopy, the experiment starts with the two atoms separated by $R=25 \mu \mathrm{m}$, aligned along the quantization axis, and prepared in the state $|\uparrow \uparrow\rangle$ after the initial Rydberg excitation pulse. The addressing laser and microwave field $\left[\Omega_{\mathrm{mw}} /(2 \pi)=0.1 \mathrm{MHz}\right]$ are then switched on during a time $\tau=\pi /\left(\sqrt{2} \Omega_{\mathrm{mw}}\right)=3.5 \mu$ s to induce a spin-flip before a final Rydberg deexcitation pulse allows the read-out of the final population in $|\uparrow \uparrow\rangle$. The experiment is repeated $\approx 100$ times for each set of parameters $\Delta_{\mathrm{mw}}, \Delta \omega_{0}$ (the latter being tuned by changing the addressing beam detuning $\Delta_{\text {addr }}$ ) and the results are shown in Fig. 2(b). The microwave resonances, seen as a drop in the final population of state $|\uparrow \uparrow\rangle$, are very well reproduced by calculations without any adjustable parameters (right panel). Notably at $\Delta \omega_{0}=0$, the dark (subradiant) state $|-\rangle$ is not coupled anymore and the bright state $|+\rangle$ energy shift measures the interaction strength $U / h=0.40 \mathrm{MHz}$.

The enhanced microwave coupling to the bright (super-radiant) state is best seen on the Rabi oscillation in Fig. 2(c). The upper panel shows a single-atom Rabi oscillation between states $|\uparrow\rangle$ and $|\downarrow\rangle$ driven at $\Omega_{\mathrm{mw}} /(2 \pi)=1.6 \mathrm{MHz}$. The lower panel shows coherent oscillations between $|\uparrow \uparrow\rangle$ and $|+\rangle$, with a measured frequency enhancement of 1.375(5), close to the expected $\sqrt{2}$. The finite contrast of these microwave-driven Rabi oscillations is due to the finite efficiency $\eta=0.88$ of the Rydberg excitation pulse, resulting directly in imperfect initial transfer of each atom in $|\uparrow\rangle$ and in small measurement errors [21]. In this experiment, the state $|\downarrow \downarrow\rangle$ is not populated as a consequence of the Rydberg blockade shifting the singly excited state $|+\rangle$.

We now use the addressing beam as a tool to freeze at will the spin-exchange dynamics between the two states $|\uparrow \downarrow\rangle$ and $|\downarrow \uparrow\rangle$. The experimental sequence is shown in Fig. 3(a) and starts as previously with two atoms excited in $|\uparrow \uparrow\rangle$. The atoms are then initialized in $|\uparrow \downarrow\rangle$ by addressing atom 1 with $\Delta \omega_{0} /(2 \pi)=4.8 \mathrm{MHz}$, while a global microwave pulse $\left[\Omega_{\mathrm{mw}} /(2 \pi)=1.3 \mathrm{MHz}\right]$ only drives the spinflip transition on atom 2. Once the addressing laser is switched off, the system evolves for a time $T$ before the 
(a)

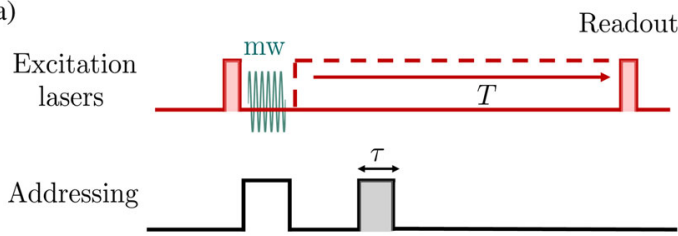

(b)

(c)
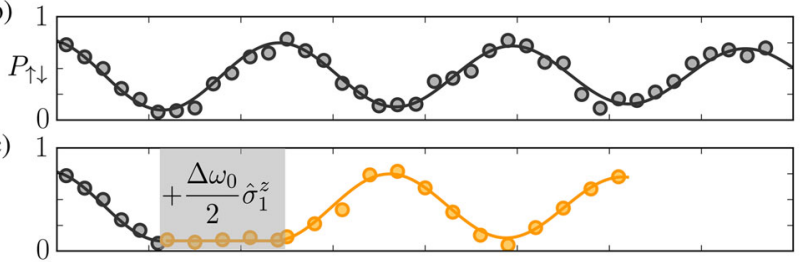

(d)

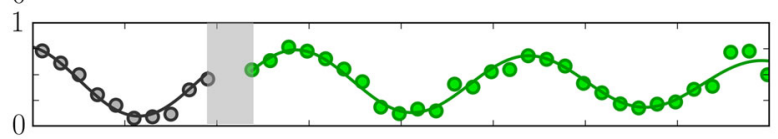

(e)

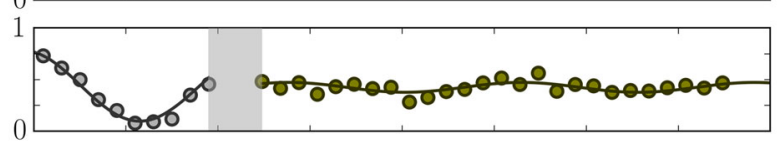

(f)

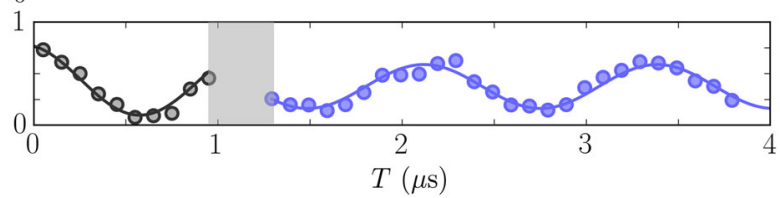

FIG. 3. (a) Experimental sequence to observe the freezing of the spin-exchange dynamics by the addressing beam with $\Delta \omega_{0} /(2 \pi)=$ $4.8 \mathrm{MHz}$ and $U / h=0.40 \mathrm{MHz}(R=25 \mu \mathrm{m})$. (b) Spin-exchange dynamics $|\uparrow \downarrow\rangle \leftrightarrow|\downarrow \uparrow\rangle$ driven by the dipole-dipole interaction at a measured frequency $2 U / h=0.80 \mathrm{MHz}$. (c) The addressing beam stops the exchange during a time $\tau=600 \mathrm{~ns}$ (gray area). [(d)-(f)] The spin exchange is frozen when $|\psi\rangle=-(1 / \sqrt{2})(|\uparrow \downarrow\rangle+i|\downarrow \uparrow\rangle)$ and the addressing energy shift imprints a relative dynamical phase $\Delta \omega_{0} \tau=2 \pi, 2.5 \pi, 3 \pi[(\mathrm{d})-(\mathrm{f})]$ between $|\uparrow \downarrow\rangle$ and $|\downarrow \uparrow\rangle$. Error bars are smaller than the symbol size. Solid lines are sinusoidal fits with fixed frequency $2 U / h$.

read-out pulse and shows coherent, interaction-driven spinexchange dynamics at a measured frequency $2 U / h=$ $0.80 \mathrm{MHz}$ as observed on the state population $P_{\uparrow \downarrow}$ in Fig. 3(b). After half a period of spin exchange, we shine again the addressing beam during a time $\tau=600 \mathrm{~ns}$ [gray area in Fig. 3(c)], thus detuning the first atom out of resonance $\left(\hbar \Delta \omega_{0} \gg U\right)$. The system is then frozen in state $|\downarrow \uparrow\rangle$, until the dynamic restarts without any noticeable loss of contrast after the addressing beam is turned off.

During the freezing time, the energy-shifted state $|\uparrow \downarrow\rangle$ acquires a dynamical phase $\phi=\Delta \omega_{0} \tau$ compared to $|\downarrow \uparrow\rangle$. To observe this relative phase, we switch on the addressing beam when the system is in the superposition of states $-(1 / \sqrt{2})(|\uparrow \downarrow\rangle+i|\downarrow \uparrow\rangle)$. During the addressing time $\tau$ it evolves into $|\psi\rangle=-(1 / \sqrt{2})\left(e^{-i \phi}|\uparrow \downarrow\rangle+i|\downarrow \uparrow\rangle\right)$ and the following spin-exchange dynamics depends on the acquired phase $\phi$. On Fig. 3(d), we adjust the addressing time $\tau$ such that $\phi=2 \pi$ and the dynamics resumes as before we froze it. For $\phi=2.5 \pi,|\psi\rangle=i|-\rangle$ is an eigenstate of the Hamiltonian and thus the populations do not evolve anymore as seen in Fig. 3(e). Finally, in Fig. 3(f), we obtain a $\pi$-phase shift on the spin-exchange dynamics when $\phi=3 \pi$. This illustrates the potential of the addressing beam for coherent manipulation of many-body quantum states.

We finally discuss the possible imperfections of this addressing technique for quantum state engineering. A first decoherence mechanism is the spontaneous emission due to the off-resonant coupling to $\left|6 P_{1 / 2}\right\rangle$, whose lifetime is $\tau_{6 P}=$ $121 \mathrm{~ns}$ [44], limiting the lifetime of the addressed state to $\tau_{\uparrow} \simeq\left(\Delta_{\mathrm{addr}} / \Omega_{\mathrm{addr}}\right)^{2} \tau_{6 P}$ [38]. For our typical parameters, $\tau_{\uparrow}$ reaches several tens of microseconds, close to the natural lifetime of the involved Rydberg levels, and thus barely affects the dynamics of the system. Another process can also bring the atom out of the spin- $1 / 2$ basis: Because of the $\sigma^{+}+\sigma^{-}$polarization of the addressing beam, both Zeeman states $|\uparrow\rangle=\left|n D_{3 / 2}, m_{J}=3 / 2\right\rangle$ and $|0\rangle=$ $\left|n D_{3 / 2}, m_{J}=-1 / 2\right\rangle$ are coupled to $\left|6 P_{1 / 2}\right\rangle$. An atom in state $|\uparrow\rangle$ can thus be transferred to $|0\rangle$ in a Raman process with effective Rabi frequency $\Omega_{\text {Raman }}=(1 / \sqrt{3}) \Omega_{\text {addr }}^{2} /$ $\left(2 \Delta_{\text {addr }}\right)=(2 / \sqrt{3}) \Delta \omega_{0}$. Because of the magnetic field, the two Zeeman levels $|\uparrow\rangle$ and $|0\rangle$ are separated in frequency by $\delta /(2 \pi)=15 \mathrm{MHz}$ and the Raman process is suppressed as long as $\Omega_{\text {Raman }} \ll \delta$. Simulations show that for the data presented here, this process gives a transfer probability of at most $\sim 8 \%$. Using a higher magnetic field, splitting even more the Zeeman structure, would be a straightforward way to reduce this transfer out of the qubit subspace. Finally, for some applications in quantum state engineering, it is important to have a fine control of the dynamical phase $\phi=\Delta \omega_{0} \tau$, which requires us to limit shot-to-shot fluctuations of the addressing beam power (the finite switching time of the addressing beam, on the order of $10 \mathrm{~ns}$, has a negligible influence for the data shown here as our addressing times are hundreds of ns). All the above imperfections can be made negligible by modest technical improvements.

In summary, we have demonstrated local selective shifts of Rydberg levels, allowing us to bring two atoms in and out of the resonant dipole-dipole interaction regime. This makes it possible to create entangled states of two atoms in different ways. A first possibility consists in starting from $|\uparrow \uparrow\rangle$ and in collectively driving the system with microwaves to $|+\rangle$ (Fig. 2). In contrast to the usual Rydberg blockade protocols, based on optical driving between $|g\rangle$ and $|\uparrow\rangle$, this approach benefits from (i) the high amplitude and phase stability of microwave sources, (ii) the long wavelength of microwave fields compared to the interatomic spacing, making motional phases negligible [45], and (iii) the fact that the entangled state $|+\rangle$ is separated by a finite energy gap from $|-\rangle$, thus decreasing its sensitivity to dephasing [46]. A second possibility is to start from $|\uparrow \downarrow\rangle$ and to stop the spin-exchange dynamics at the appropriate time (Fig. 3), which allows us to create any coherent superposition of $|\uparrow \downarrow\rangle$ and $|\downarrow \uparrow\rangle$, in particular, the otherwise inaccessible dark subradiant state $|-\rangle$. In future work, one could even generate several independently controlled 
addressing beams using spatial and/or temporal light modulators. Then, such a system would open exciting prospects for quantum state engineering (one could, e.g., straightforwardly initialize a one-dimensional chain in the state $|\uparrow \downarrow \uparrow \downarrow \uparrow \downarrow \cdots\rangle$, or more complex ones, and then let it evolve), for quantum state tomography (one could, e.g., address, with a different light shift, two selected atoms in an array, thus freezing interactions, both between them and with the other spins, such that independent single-spin rotations followed by measurements of $\sigma_{z}$ would allow reconstructing the arbitrary spin-spin correlations needed for tomography), and for quantum simulation of the $X Y$ model using arrays of single Rydberg atoms (one could e.g., apply a smooth, spatially varying longitudinal field for focusing initially delocalized spin excitations onto a single spin [47], or apply controlled disorder on the spins for many-body localization studies [5,7]).

This work benefited from financial support by the EU (Grant No. H2020 FET-PROACT Project RySQ), by the PALM Labex (projects QUANTICA and XYLOS) and by the Région Île-de-France in the framework of DIM Nano-K.

S. de L., D. B., and V.L. contributed equally to this work.

[1] I. M. Georgescu, S. Ashhab, and F. Nori, Rev. Mod. Phys. 86, 153 (2014).

[2] R. Blatt and D. Wineland, Nature (London) 453, 1008 (2008).

[3] M. Veldhorst, C. H. Yang, J. C. C. Hwang, W. Huang, J. P. Dehollain, J. T. Muhonen, S. Simmons, A. Laucht, F. E. Hudson, K. M. Itoh, A. Morello, and A. S. Dzurak, Nature (London) 526, 410 (2015).

[4] Y. Salathé, M. Mondal, M. Oppliger, J. Heinsoo, P. Kurpiers, A. Potočnik, A. Mezzacapo, U. Las Heras, L. Lamata, E. Solano, S. Filipp, and A. Wallraff, Phys. Rev. X 5, 021027 (2015).

[5] J.-Y. Choi, S. Hild, J. Zeiher, P. Schauss, A. Rubio-Abadal, T. Yefsah, V. Khemani, D. A. Huse, I. Bloch, and C. Gross, Science 352, 1547 (2016).

[6] M. Marcuzzi, J. Minář, D. Barredo, S. de Léséleuc, H. Labuhn, T. Lahaye, A. Browaeys, E. Levi, and I. Lesanovsky, Phys. Rev. Lett. 118, 063606 (2017)

[7] J. Smith, A. Lee, P. Richerme, B. Neyenhuis, P. W. Hess, P. Hauke, M. Heyl, D. A. Huse, and C. Monroe, Nat. Phys. 12, 907 (2016).

[8] M. Veldhorst, J. C. C. Hwang, C. H. Yang, A. W. Leenstra, B. de Ronde, J. P. Dehollain, J. T. Muhonen, F. E. Hudson, K. M. Itoh, A. Morello, and A. S. Dzurak, Nat. Nanotechnol. 9, 981 (2014).

[9] A. A. Houck, H. E. Türeci, and J. Koch, Nat. Phys. 8, 292 (2012).

[10] C. Weitenberg, M. Endres, J. F. Sherson, M. Cheneau, P. Schauss, T. Fukuhara, I. Bloch, and S. Kuhr, Nature (London) 471, 319 (2011).
[11] H. Labuhn, S. Ravets, D. Barredo, L. Béguin, F. Nogrette, T. Lahaye, and A. Browaeys, Phys. Rev. A 90, 023415 (2014).

[12] T. Xia, M. Lichtman, K. Maller, A. W. Carr, M. J. Piotrowicz, L. Isenhower, and M. Saffman, Phys. Rev. Lett. 114, 100503 (2015).

[13] Y. Wang, X. Zhang, T. A. Corcovilos, A. Kumar, and D. S. Weiss, Phys. Rev. Lett. 115, 043003 (2015).

[14] M. Saffman, T. G. Walker, and K. Mølmer, Rev. Mod. Phys. 82, 2313 (2010).

[15] H. Weimer, M. Muller, I. Lesanovsky, P. Zoller, and H. P. Büchler, Nat. Phys. 6, 382 (2010).

[16] A. Browaeys, D. Barredo, and T. Lahaye, J. Phys. B 49, 152001 (2016).

[17] J. Zeiher, R. van Bijnen, P. Schauss, S. Hild, J.-Y. Choi, T. Pohl, I. Bloch, and C. Gross, Nat. Phys. 12, 1095 (2016).

[18] Y.-Y. Jau, A. M. Hankin, T. Keating, I. H. Deutsch, and G. W. Biedermann, Nat. Phys. 12, 71 (2016).

[19] D. Barredo, S. de Léséleuc, V. Lienhard, T. Lahaye, and A. Browaeys, Science 354, 1021 (2016).

[20] M. Endres, H. Bernien, A. Keesling, H. Levine, E. R. Anschuetz, A. Krajenbrink, C. Senko, V. Vuletic, M. Greiner, and M. D. Lukin, Science 354, 1024 (2016).

[21] H. Labuhn, D. Barredo, S. Ravets, S. de Léséleuc, T. Macrì, T. Lahaye, and A. Browaeys, Nature (London) 534, 667 (2016).

[22] X. L. Deng, D. Porras, and J. I. Cirac, Phys. Rev. A 72, 063407 (2005).

[23] P. Hauke, F. M. Cucchietti, A. Müller-Hermes, M. C. Bañuls, J. I. Cirac, and M. Lewenstein, New J. Phys. 12, 113037 (2010).

[24] C. N. Varney, K. Sun, V. Galitski, and M. Rigol, Phys. Rev. Lett. 107, 077201 (2011).

[25] D. Peter, S. Müller, S. Wessel, and H. P. Büchler, Phys. Rev. Lett. 109, 025303 (2012).

[26] B. Yan, S. Moses, B. Gadway, J. Covey, K. Hazzard, A. Rey, D. Jin, and J. Ye, Nature (London) 501, 521 (2013).

[27] M. Dalmonte, S. I. Mirzaei, P. R. Muppalla, D. Marcos, P. Zoller, and G. Kirchmair, Phys. Rev. B 92, 174507 (2015).

[28] I. Mourachko, D. Comparat, F. de Tomasi, A. Fioretti, P. Nosbaum, V. M. Akulin, and P. Pillet, Phys. Rev. Lett. 80, 253 (1998).

[29] W. R. Anderson, J. R. Veale, and T. F. Gallagher, Phys. Rev. Lett. 80, 249 (1998).

[30] D. Barredo, S. Ravets, H. Labuhn, L. Béguin, A. Vernier, F. Nogrette, T. Lahaye, and A. Browaeys, Phys. Rev. Lett. 112, 183002 (2014).

[31] D. Maxwell, D. J. Szwer, D. Paredes-Barato, H. Busche, J. D. Pritchard, A. Gauguet, M. P. A. Jones, and C. S. Adams, Phys. Rev. A 89, 043827 (2014).

[32] A. P. Orioli, A. Signoles, H. Wildhagen, G. Günter, J. Berges, S. Whitlock, and M. Weidemüller, arXiv:1703.05957.

[33] The $C_{6}$ coefficients for pair states constructed from the states $61 D_{3 / 2}$ and $62 P_{1 / 2}$ are $C_{6, d d} \simeq-762 \mathrm{GHz} \mu \mathrm{m}^{6}$ and $C_{6, p p} \simeq-20 \mathrm{GHz} \mu \mathrm{m}^{6}$ [36,37]. For $R=25 \mu \mathrm{m}$ used in most of the experiments reported here, this gives van der Waals interactions of only a few $\mathrm{kHz}$.

[34] L. Béguin, A. Vernier, R. Chicireanu, T. Lahaye, and A. Browaeys, Phys. Rev. Lett. 110, 263201 (2013).

[35] F. Nogrette, H. Labuhn, S. Ravets, D. Barredo, L. Béguin, A. Vernier, T. Lahaye, and A. Browaeys, Phys. Rev. X 4, 021034 (2014). 
[36] N. Šibalić, J. D. Pritchard, C.S. Adams, and K. J. Weatherill, arXiv:1612.05529.

[37] S. Weber, C. Tresp, H. Menke, A. Urvoy, O. Firstenberg, H. P. Büchler, and S. Hofferberth, J. Phys. B 50, 133001 (2017).

[38] M. Saffman and T. G. Walker, Phys. Rev. A 72, 022347 (2005).

[39] L. Li, Y. O. Dudin, and A. Kuzmich, Nature (London) 498 , 466 (2013).

[40] The beam waist was determined by using the addressing beam as a single-atom trap. The trap depth is measured by Rydberg spectroscopy [11] and the trapping frequency by parametric heating [41].
[41] M. J. Piotrowicz, M. Lichtman, K. Maller, G. Li, S. Zhang, L. Isenhower, and M. Saffman, Phys. Rev. A 88, 013420 (2013). [42] High Finesse, WLM SU10.

[43] K. C. Younge, B. Knuffman, S. E. Anderson, and G. Raithel, Phys. Rev. Lett. 104, 173001 (2010).

[44] E. Gomez, S. Aubin, L. A. Orozco, and G. D. Sprouse, J. Opt. Soc. Am. B 21, 2058 (2004).

[45] T. Wilk, A. Gaëtan, C. Evellin, J. Wolters, Y. Miroshnychenko, P. Grangier, and A. Browaeys, Phys. Rev. Lett. 104, 010502 (2010).

[46] D. Viscor (to be published).

[47] A. W. Glaetzle, K. Ender, D. Wild, S. Choi, H. Pichler, M. Lukin, and P. Zoller, arXiv:1704.08837. 